Article

\title{
The Weitzenböck Type Curvature Operator for Singular Distributions
}

\author{
Paul Popescu ${ }^{1, *}$ (i), Vladimir Rovenski ${ }^{2}$ (i) and Sergey Stepanov ${ }^{3}$ (i) \\ 1 Department of Applied Mathematics, University of Craiova, Str. Al. Cuza, No, 13, 200585 Craiova, Romania \\ 2 Department of Mathematics, University of Haifa, Mount Carmel, 31905 Haifa, Israel; \\ vrovenski@univ.haifa.ac.il \\ 3 Russian Institute for Scientific and Technical Information of the Russian Academy of Sciences, \\ 125190 Moscow, Russia; s.e.stepanov@mail.ru \\ * Correspondence: paul_p_popescu@yahoo.com
}

Received: 16 February 2020; Accepted: 3 March 2020; Published: 6 March 2020

check for updates

\begin{abstract}
We study geometry of a Riemannian manifold endowed with a singular (or regular) distribution, determined as an image of the tangent bundle under smooth endomorphisms. Following construction of an almost Lie algebroid on a vector bundle, we define the modified covariant and exterior derivatives and their $L^{2}$ adjoint operators on tensors. Then, we introduce the Weitzenböck type curvature operator on tensors, prove the Weitzenböck type decomposition formula, and derive the Bochner-Weitzenböck type formula. These allow us to obtain vanishing theorems about the null space of the Hodge type Laplacian. The assumptions used in the results are reasonable, as illustrated by examples with $f$-manifolds, including almost Hermitian and almost contact ones.
\end{abstract}

Keywords: Riemannian manifold; singular distribution; Weitzenböck curvature operator; Hodge Laplacian; almost Lie algebroid

MSC: 53C15; 53C21

\section{Introduction}

Distributions, as subbundles of the tangent bundle on a manifold, arise in such topics of mathematics and physics as fiber bundles, Lie groups actions, almost contact, Poisson and sub-Riemannian manifolds, e.g., [1-5]. Lie algebroids are generalizations of Lie algebras and integrable regular distributions, a more general concept of an almost Lie algebroid, i.e., an anchored vector bundle with an almost Lie bracket, is involved in nonholonomic geometry, e.g., [6-8].

Singularities play a crucial role in mathematics (e.g., in real and complex analysis, algebraic geometry and differential topology, theory of dynamical systems), and its applications in natural and technical sciences (e.g., in theories of nonlinear control systems and relativity). There is definite interest of pure and applied mathematicians, e.g., [3,9], to singular distributions and foliations, i.e., having varying dimension: just mention (singular) Riemannian foliations, i.e., every geodesic that is perpendicular at one point to a leaf remains perpendicular to every leaf it meets; an example is the orbital decomposition of the isometric actions of a Lie group. A singular distribution $\mathcal{D}$ on a manifold $M$ assigns to each point $x \in M$ a linear subspace $\mathcal{D}_{x}$ of the tangent space $T_{x} M$ in such a way that, for any $v \in \mathcal{D}_{x}$, there exists a smooth vector field $V$ defined in a neighborhood $U$ of $x$ and such that $V(x)=v$ and $V(y) \in \mathcal{D}_{y}$ for all $y$ of $U$. A priori, the dimension $\operatorname{dim} \mathcal{D}_{x}$ depends on $x \in M$. If $\operatorname{dim} \mathcal{D}_{x}=$ const, then we obtain a regular distribution. Singular foliations are defined as families of maximal integral submanifolds (leaves) of integrable generalized distributions (certainly, regular foliations correspond to integrable regular distributions). The study of singular distributions is important also because there 
are plenty of manifolds that exist that do not admit smooth (codimension-one) distributions, while all of them admit such distributions defined outside some "set of singularities".

Let $M$ be a connected smooth $n$-dimensional manifold, $g=\langle\cdot, \cdot\rangle$ is a Riemannian metric on $M$, $T M$ is the tangent bundle, $\mathcal{X}_{M}$ is the Lie algebra of smooth vector fields on $M$, and End $(T M)$ are smooth endomorphisms of TM onto itself, i.e., linear maps on the fibers of TM.

Here, we determine singular distributions as images of TM with smooth endomorphisms.

Definition 1 (see [10]). An image $\mathcal{D}=P(T M)$ of a smooth endomorphism $P \in \operatorname{End}(T M)$ will be called a generalized vector subbundle of TM or a singular distribution.

Example 1. (a) Consider $P \in \operatorname{End}\left(T \mathbb{R}^{n}\right)$ having as image $\mathcal{D}=P\left(T \mathbb{R}^{n}\right)$ the singular distribution tangent to spheres centered at the origin and the origin itself as a singular point. Namely, $\mathcal{D}$ is spanned by the vector fields $Z_{i j}=x^{j} \partial_{i}-x^{i} \partial_{j}$ for $1 \leq i<j \leq n$. For $n=2$ and coordinates $(x, y)$, set $P(x, y ; X, Y)=$ $(x, y ; y(y X-x Y),-x(y X-x Y))$. Then, the vector fields $P(x, y ; 1,0)$ and $P(x, y ; 0,1)$ generate $\mathcal{D}$. The case of $n>2$ is similar.

(b) Let $P \in \operatorname{End}(T M)$ on $(M, g)$ be of constant rank and satisfying conditions

$$
P^{2}=P, \quad P^{*}=P,
$$

where $P^{*}$ is adjoint endomorphism to $P$, i.e., $\left\langle P^{*} X, Y\right\rangle=\langle X, P Y\rangle$, then we have an almost-product structure on $(M, g)$, see [11]. In this case, $P$ and $\tilde{P}=\mathrm{id}-P$ (that can be orthoprojectors) determine complementary orthogonal regular distributions $P(T M)=\mathcal{D}$ and $\tilde{P}(T M)=\widetilde{\mathcal{D}}$, neither of which is in general integrable. Twisted (and warped) products give us popular examples of foliations (integrable regular distributions), e.g., [12,13]. Furthermore, given a Riemannian metric $g$ and a singular distribution $\mathcal{D}$ on $M$, there are self-adjoint endomorphisms $P$ and $\tilde{P}$ of $T M$ onto itself such that $P(T M)=\mathcal{D}$ and $\tilde{P}(T M)=\widetilde{\mathcal{D}}$ are smooth orthogonal distributions and pointwise orthogonal sum decomposition $T M=P(T M) \oplus \tilde{P}(T M)$ holds on a dense subset of $M$ (see [14]).

The Hodge Laplacian $\Delta_{H}=d \delta+\delta d$, where $d$ is the exterior differential operator, and $\delta$ is its adjoint operator with respect to the $L^{2}$ inner product, can be decomposed into two terms,

$$
\Delta_{H}=\bar{\Delta}+\mathcal{R} i c
$$

One is the connection Laplacian $\bar{\Delta}=\nabla^{*} \nabla$, and the second term (depends linearly on the Riemannian curvature tensor) is called the Weitzenböck curvature operator on $(0, k)$-tensors $S$ over $(M, g)$,

$$
\begin{aligned}
\mathcal{R i c} & (S)\left(X_{1}, \ldots, X_{k}\right)=\sum_{\alpha=1}^{k} \sum_{i=1}^{n}\left(R_{e_{i}, X_{\alpha}} S\right)(\underbrace{X_{1}, \ldots, e_{i}}_{\alpha}, \ldots, X_{k}) \\
& =-2 \sum_{i, m, a ; b<a} R\left(e_{i}, X_{a}, e_{m}, X_{b}\right) \cdot S(\underbrace{X_{1}, \ldots, e_{m}}_{b}, \ldots, \underbrace{e_{i}}_{a-b}, \ldots, X_{k}) \\
& +\sum_{i, a} \operatorname{Ric}\left(e_{i}, X_{a}\right) \cdot S(\underbrace{X_{1}, \ldots, e_{i}}_{a}, \ldots, X_{k}),
\end{aligned}
$$

or, in coordinates, $\mathcal{R} i c(S)_{i_{1}, \ldots, i_{k}}=-2 \sum_{a<b} R_{j i_{a} p i_{b}} S_{i_{1} \ldots \ldots \ldots i_{k}}+\sum_{a} \operatorname{Ric}_{i_{a} j} S_{i_{1} \ldots \ldots i_{k}}{ }^{j}$ e.g., [15]. Note that $\mathcal{R} i c$ is the Ricci tensor when evaluated on vector fields and 1 -forms. Here, and in what follows, $\left(e_{i}\right)$ is a local orthonormal frame on TM. Formula (2) is important in the study of interactions between the geometry and topology of manifolds. The Bochner technique works for skew-symmetric tensors lying in the kernel of $\Delta_{H}$ (or its generalization $\Delta_{L}=\bar{\Delta}+c \mathcal{R}$ ic for any $c \neq 0$, called the Lichnerowicz Laplacian, which acts on $(0, k)$-tensors) on a closed manifold, e.g., [15-18]: using maximum principles, they prove that such tensors are parallel. For this, we need $\langle\mathcal{R i c}(S), S\rangle \geq 0$ (where $\langle\cdot, \cdot\rangle$ is the scalar product defined on tensor bundles over $M$ by the metric), which by (2) is equivalent to inequality 
$\langle\bar{\Delta} S, S\rangle \leq 0$. There is a simple formula for $\operatorname{Ric}(S)$, which shows that $\langle\operatorname{Ric}(S), S\rangle \geq 0$ when the curvature operator on $\Lambda^{2}(T M)$ is non-negative [15,19].

The work is devoted to the differential geometry of a Riemannian manifold $(M, g)$ endowed with a singular (or regular) distribution $P(T M)$ and continues our study $[7,8,10,14,20]$. In Section 2, we recall the construction of an almost Lie algebroid on a vector bundle. Following this construction, in Sections 3 and 4, we define the derivatives $\nabla^{P}$ and $d^{P}$ and their $L^{2}$ adjoint operators on tensors. The assumptions that we use in the results are reasonable, as illustrated in Section 3 by examples with metric $f$-manifolds, including almost Hermitian and almost contact manifolds. In Section 5 , using $\nabla^{P}$ and making some assumptions about $P$ (which are trivial when $P=\mathrm{id}_{T M}$ ), we define the curvature operator $R^{P}$ and the Weitzenböck type curvature operator on tensors. Then, we prove the Weitzenböck type decomposition formula and derive the Bochner-Weitzenböck type formula. In Section 7 (see also Section 4), we obtain vanishing results, Theorem 3 and Corollary 1, about the null space of the Hodge type Laplacian. Appendix A contains the proof of Proposition 6.

\section{The Almost Lie Algebroid Structure}

Let $\pi_{E}: E \rightarrow M$ be a smooth vector bundle of rank $k$ over $M$ (i.e., a smooth fibre bundle with fibre $\left.\mathbb{R}^{k}\right)$. A Riemannian bundle $(E, g)$ over $M$ has a symmetric positive definite $(0,2)$-tensor field $g=\langle\cdot, \cdot\rangle$, i.e., $g_{x}$ is an inner product in each fiber $E_{x}$ smoothly depending on $x \in M$. The main example is $E=T M$ with the Riemannian structure (metric) on $M$, see more examples in [21].

Recall some facts about another important structure on $E$, see, e.g., $[6,7,20]$.

Definition 2. An anchor on $E$ is a morphism $\rho: E \rightarrow T M$ of vector bundles. A skew-symmetric bracket on $E$ is a map $[\cdot, \cdot]_{\rho}: \mathfrak{X}_{E} \times \mathfrak{X}_{E} \rightarrow \mathfrak{X}_{E}$ such that

$$
[Y, X]_{\rho}=-[X, Y]_{\rho}, \quad[X, f Y]_{\rho}=\rho(X)(f) Y+f[X, Y]_{\rho}, \quad \rho\left([X, Y]_{\rho}\right)=[\rho(X), \rho(Y)]
$$

for all $X, Y \in \mathfrak{X}_{E}$ and $f \in C^{\infty}(M)$. The anchor and the skew-symmetric bracket give an almost Lie algebroid structure on a vector bundle $E$. The tensor map $\mathcal{J}_{\rho}: \mathfrak{X}_{E} \times \mathfrak{X}_{E} \times \mathfrak{X}_{E} \rightarrow \mathfrak{X}_{E}$, given by

$$
\mathcal{J}_{\rho}(X, Y, Z)=\sum_{\text {circ. }}\left[X,[Y, Z]_{\rho}\right]_{\rho}
$$

is called the Jacobiator of the bracket; using $(4)_{3}$, we get $\rho \mathcal{J}_{\rho}=0$. An almost Lie algebroid is a Lie algebroid provided that the Jacobiator of the bracket $[\because, \cdot]_{\rho}$ vanishes.

Note that axiom $(4)_{3}$ means vanishing of the following operator:

$$
\mathfrak{D}^{\rho}(X, Y)=[\rho X, \rho Y]-\rho\left([X, Y]_{\rho}\right)
$$

There is a bijective correspondence between almost Lie algebroid structures on $E$ and the exterior differentials of the exterior algebra $\Lambda(E)=\bigoplus_{k \in \mathbb{N}} \Lambda^{k}(E)$ of the dual bundle $E^{*}$, see [8]; here $\Lambda^{k}(E)$ is the set of $k$-forms over $E$. The exterior differential $d^{\rho}$, corresponding to the almost Lie algebroid structure $\left(E, \rho,[\cdot, \cdot]_{\rho}\right)$, is given by

$$
\begin{aligned}
d^{\rho} \omega\left(X_{0}, \ldots, X_{k}\right) & =\sum_{i=0}^{k}(-1)^{i}\left(\rho X_{i}\right)\left(\omega\left(X_{0}, \ldots, \widehat{X}_{i}, \ldots, X_{k}\right)\right) \\
& +\sum_{0 \leq i<j \leq k}(-1)^{i+j} \omega\left(\left[X_{i}, X_{j}\right]_{\rho}, X_{0}, \ldots, \widehat{X}_{i}, \ldots, \widehat{X}_{j}, \ldots, X_{k}\right),
\end{aligned}
$$

where $X_{0}, \ldots, X_{k} \in \mathfrak{X}_{E}$ and $\omega \in \Lambda^{k}(E)$ for $k \geq 0$. For $k=0$, we have

$$
d^{\rho} f(X)=(\rho X)(f), \quad X \in \mathfrak{X}_{E}, f \in C^{\infty}(M)=\Lambda^{0}(E) .
$$


Recall that a skew-symmetric algebroid defines uniquely an exterior differential $d^{\rho}$ on $\Lambda(T M)$, and it gives rise to (see also Proposition 5)

- a skew-symmetric algebroid if and only if $\left(d^{\rho}\right)^{2} f=0$ for $f \in C^{\infty}(M)$;

- a Lie algebroid if and only if $\left(d^{\rho}\right)^{2} f=0$ and $\left(d^{\rho}\right)^{2} \omega=0$ for $f \in C^{\infty}(M)$ and $\omega \in \Lambda^{1}(T M)$.

Definition 3. A $\rho$-connection on $(E, \rho)$ is a map $\nabla^{\rho}: \mathfrak{X}_{E} \times \mathcal{X}_{E} \rightarrow \mathfrak{X}_{E}$ satisfying Koszul conditions

$$
\nabla_{X}^{\rho}(f Y+Z)=\rho(X)(f) Y+f \nabla_{X}^{\rho} Y+\nabla_{X}^{\rho} Z, \quad \nabla_{f X+Z}^{\rho} Y=f \nabla_{X}^{\rho} Y+\nabla_{Z}^{\rho} Y .
$$

For a $\rho$-connection $\nabla^{\rho}$ on $E$, they define torsion $T^{\rho}: \mathfrak{X}_{E} \times \mathcal{X}_{E} \rightarrow \mathfrak{X}_{E}$ and curvature $R^{\rho}: \mathfrak{X}_{E} \times \mathcal{X}_{E} \times \mathcal{X}_{E} \rightarrow \mathfrak{X}_{E}$ by "usual" formulas

$$
\begin{aligned}
& T^{\rho}(X, Y)=\nabla_{X}^{\rho} Y-\nabla_{Y}^{\rho} X-[X, Y]_{\rho} \\
& R_{X, Y}^{\rho} Z=\nabla_{X}^{\rho} \nabla_{Y}^{\rho} Z-\nabla_{Y}^{\rho} \nabla_{X}^{\rho} Z-\nabla_{[X, Y]_{\rho}}^{\rho} Z .
\end{aligned}
$$

The following equality holds, see [7]:

$$
\sum_{\text {circ. }} R_{X, Y}^{\rho} Z=\sum_{\text {circ. }}\left[\left(\nabla_{X}^{\rho} T^{\rho}\right)(Y, Z)+T^{\rho}\left(T^{\rho}(X, Y), Z\right)\right]+\mathcal{J}_{P}(X, Y, Z) .
$$

We will apply the almost Lie algebroid structure to singular distributions on $M$, namely, in the rest of paper, we assume $E=T M$ and $\rho=P \in \operatorname{End}(T M)$, see Definition 1.

\section{The Modified Covariant Derivative and Its $L^{2}$-Adjoint}

The $L^{2}$-scalar product on compactly supported $C^{\infty}$-sections of $T M$ and the Hilbert space $L^{2}(T M)$ are defined by $(u, v)=\int_{M}\langle u, v\rangle \mathrm{d} \mathrm{vol}_{g}$, where $\mathrm{d}$ vol is the volume form of $g$. Using the $L^{2}$-structure on $C^{\infty}(T M)$, they define the connection Laplacian by $\bar{\Delta}=\nabla^{*} \nabla$, where $\nabla^{*}$ is the $L^{2}$-adjoint of the Levi-Civita connection $\nabla$.

The covariant derivative of a $(m, k)$-tensor $S$ on $T M$ is a $(m, k+1)$-tensor, e.g., [15],

$$
\begin{aligned}
(\nabla S)\left(Y, X_{1}, \ldots, X_{k}\right) & =\left(\nabla_{Y} S\right)\left(X_{1}, \ldots, X_{k}\right) \\
& =\nabla_{Y}\left(S\left(X_{1}, \ldots, X_{k}\right)\right)-\sum_{i \leq k} S\left(X_{1}, \ldots, \nabla_{Y} X_{i}, \ldots, X_{k}\right),
\end{aligned}
$$

where $Y, X_{i} \in \mathcal{X}_{M}$. Its $L^{2}$-adjoint is given by

$$
\left(\nabla^{*} S\right)\left(X_{2}, \ldots, X_{k}\right)=-\sum_{i}\left(\nabla_{e_{i}} S\right)\left(e_{i}, X_{2}, \ldots, X_{k}\right) .
$$

The divergence of a $(0, k+1)$-tensor $S$ is a $(0, k)$-tensor $\operatorname{div} S=-\nabla^{*} S$. Using a metric $g$ on a manifold, they identify $(1, k)$-tensors with $(0, k+1)$-tensors: $S^{j}{ }_{i_{1}, \ldots i_{k}}=g^{m j} S_{m, i_{1}, \ldots i_{k}}$. Thus, for a $(1, k)$-tensor $S$, we can equivalently write $\operatorname{div} S=\operatorname{trace}\left(Y \rightarrow \nabla_{Y} S\right)$, that is,

$$
(\operatorname{div} S)\left(X_{1}, \ldots, X_{k}\right)=\sum_{i}\left\langle\left(\nabla_{i} S\right)\left(X_{1}, \ldots, X_{k}\right), e_{i}\right\rangle,
$$

or $(\operatorname{div} S)_{i_{1}, \ldots i_{k}}=\nabla_{j} S^{j}{ }_{i_{1}, \ldots i_{k}}$ in coordinates. For example, if $S$ is a $(1,1)$-tensor, then $\nabla^{*} S$ is a vector field and $\operatorname{div} S$ is a 1 -form. For the divergence $\operatorname{div} X=\operatorname{trace}\left(Y \rightarrow \nabla_{Y} X\right)$ of a vector field $X \in \mathcal{X}_{M}$, we have $\operatorname{div} X=-\nabla^{*} X^{b}$, where $X^{b}$ is the 1 -form dual to $X$. The divergence of a vector field can also be defined by

$$
d\left(\iota_{X} d \mathrm{vol}\right)=(\operatorname{div} X) d \mathrm{vol},
$$

If either $X$ has compact support or $M$ is closed, then the Divergence Theorem reads as

$$
\int_{M}(\operatorname{div} X) \mathrm{d} \operatorname{vol}_{g}=0
$$


Given $P \in \operatorname{End}(T M)$, define a P-connection $\nabla^{P}: \mathcal{X}_{M} \times \mathcal{X}_{M} \rightarrow \mathcal{X}_{M}$ (see Definition 3, which generally is not a linear connection on $T M$ ) by

$$
\nabla_{X}^{P} Y:=\nabla_{P X} Y \quad\left(X, Y \in \mathcal{X}_{M}\right),
$$

and set $\nabla_{X}^{P} f=(P X) f$ for $f \in C^{\infty}(M)$. We will use $\nabla^{P}$ to define the modified covariant derivative on tensors and its $L^{2}$-adjoint on $(M, g)$ endowed with a singular distribution.

Definition 4. The P-covariant derivative on tensors is defined by the following:

-If $f \in C^{\infty}(M)$, then $\left(\nabla^{P} f\right)(X)=\nabla_{X}^{P} f$, and $\nabla^{P} f$ is called the P-gradient of $f$.

- If $Y \in \mathcal{X}_{M}$, then $\left(\nabla^{P} Y\right)(X)=\nabla_{X}^{P} Y$, see (12).

-If $S$ is a $(s, k)$-tensor, where $s, k=0,1$, then

$$
\left(\nabla^{P} S\right)\left(Y, X_{1}, \ldots, X_{k}\right)=\nabla_{Y}^{P}\left(S\left(X_{1}, \ldots, X_{k}\right)\right)-\sum_{i=1}^{k} S\left(X_{1}, \ldots, \nabla_{Y}^{P} X_{i}, \ldots, X_{k}\right) .
$$

- This makes sense for all $s, k \geq 0$, using the product rule with respect to tensors:

$$
\nabla_{X}^{P}\left(S_{1} \otimes S_{2}\right)=\nabla_{X}^{P} S_{1} \otimes S_{2}+S_{1} \otimes \nabla_{X}^{P} S_{2}
$$

If $\nabla^{P} S=0$, then the tensor $S$ is called P-parallel.

Notice that the metric and volume form are $P$-parallel, since $\nabla^{P}$ has the metric property:

$$
\nabla_{X}^{P}\langle Y, Z\rangle=\left\langle\nabla_{P X} Y, Z\right\rangle+\left\langle Y, \nabla_{P X} Z\right\rangle=\left\langle\nabla_{X}^{P} Y, Z\right\rangle+\left\langle Y, \nabla_{X}^{P} Z\right\rangle
$$

for all $X, Y, Z \in \mathfrak{X}_{M}$, and commutes with contractions for tensors: $\nabla_{X}^{P}(\operatorname{Tr} S)=\operatorname{Tr}\left(\nabla_{X}^{P} S\right)$.

The following pointwise inner products and norms for $(0, k)$-tensors will be used:

$$
\left\langle S_{1}, S_{2}\right\rangle=\sum_{i_{1}, \ldots, i_{k}} S_{1}\left(e_{i_{1}}, \ldots, e_{i_{k}}\right) S_{2}\left(e_{i_{1}}, \ldots, e_{i_{k}}\right), \quad\|S\|=\sqrt{\langle S, S\rangle}
$$

with respect to any local orthonormal frame $\left\{e_{i}\right\}$ while, for $k$-forms, we set

$$
\left\langle\omega_{1}, \omega_{2}\right\rangle=\sum_{i_{1}<\ldots<i_{k}} \omega_{1}\left(e_{i_{1}}, \ldots, e_{i_{k}}\right) \omega_{2}\left(e_{i_{1}}, \ldots, e_{i_{k}}\right) .
$$

The next proposition generalizes ([15], Proposition 2.2.8) and shows that, under certain conditions, the $L^{2}$-adjoint to the P-covariant derivative on tensors, denoted here by $\nabla^{* P}$, is given by

$$
\left(\nabla^{* P} S\right)\left(X_{2}, \ldots, X_{k}\right)=-\sum_{i}\left(\nabla_{e_{i}}^{P} S\right)\left(e_{i}, X_{2}, \ldots, X_{k}\right) .
$$

Lemma 1 (see Proposition 2.4 in [10]). Given $P \in \operatorname{End}(T M)$, condition

$$
\operatorname{div}\left(P P^{*}\right)=0
$$

is equivalent to the following one:

$$
\operatorname{div}_{P} X=\operatorname{div}\left(P P^{*} X\right), \quad X \in \mathcal{X}_{M}
$$

Condition (16) means that $\left(\operatorname{div}_{P} X\right) d$ vol is an exact form: using (10), we have

$$
\left(\operatorname{div}_{P} X\right) d \mathrm{vol}=\left(\operatorname{div}\left(P P^{*} X\right)\right) d \mathrm{vol}=d\left(\iota_{P P^{*} X} d \mathrm{vol}\right) .
$$


Thus, Lemma 1 allows us to extend the Divergence Theorem, see (11): if (15) holds and either X has compact support or $M$ is closed, then

$$
\int_{M}\left(\operatorname{div}_{P} X\right) \mathrm{d} \operatorname{vol}_{g}=0 .
$$

Proposition 1. If condition (15) holds, then $\nabla^{* P}$ is $L^{2}$-adjoint to $\nabla^{P}$ on tensors; namely, for any compactly supported $(s, t)$-tensor $S$ and $(s, t+1)$-tensor $T$, we have

$$
\left(\nabla^{* P} S_{2}, S_{1}\right)=\left(S_{2}, \nabla^{P} S_{1}\right)
$$

Proof. Define a compactly supported 1-form $\omega$ by

$$
\omega(Y)=\left\langle\iota_{Y} T, S\right\rangle, \quad Y \in \mathcal{X}_{M}
$$

Take an orthonormal frame $\left(e_{i}\right)$ such that $\nabla_{Y} e_{i}=0$ for all $Y \in T_{x} M$. To simplify calculations, assume that $s=t=1$, then (similarly to ([15], Proposition 2.2.8)) at $x \in M$,

$$
\begin{aligned}
-\nabla^{* P} \omega & =\sum_{i, j}\left\langle P e_{j}, e_{i}\right\rangle\left(\nabla_{e_{i}} \omega\right)\left(e_{j}\right)=\sum_{i, j, c}\left\langle P e_{j}, e_{i}\right\rangle \nabla_{e_{i}}\left\langle S_{2}\left(e_{j}, e_{c}\right), S_{1}\left(e_{c}\right)\right\rangle \\
& =\sum_{i, j, c}\left\langle P e_{j}, e_{i}\right\rangle\left(\left\langle\nabla_{e_{i}} S_{2}\left(e_{j}, e_{c}\right), S_{1}\left(e_{c}\right)\right\rangle+\left\langle S_{2}\left(e_{j}, e_{c}\right), \nabla_{e_{i}} S_{1}\left(e_{c}\right)\right\rangle\right) \\
& =\sum_{i, j, c}\left(\left\langle\left\langle P e_{j}, e_{i}\right\rangle \nabla_{e_{i}} S_{2}\left(e_{j}, e_{c}\right), S_{1}\left(e_{c}\right)\right\rangle+\left\langle S_{2}\left(e_{j}, e_{c}\right),\left\langle P e_{j}, e_{i}\right\rangle \nabla_{e_{i}} S_{1}\left(e_{c}\right)\right\rangle\right) \\
& =\sum_{j, c}\left(\left\langle\nabla_{e_{j}}^{P} S_{2}\left(e_{j}, e_{c}\right), S_{1}\left(e_{c}\right)\right\rangle+\left\langle S_{2}\left(e_{j}, e_{c}\right), \nabla_{e_{j}}^{P} S_{1}\left(e_{c}\right)\right\rangle\right) \\
& =-\left\langle\nabla^{* P} S_{2}, S_{1}\right\rangle+\left\langle S_{2}, \nabla^{P} S_{1}\right\rangle .
\end{aligned}
$$

The $\nabla^{* P}$ is related to the $P$-divergence $\operatorname{div}_{P} X=\operatorname{trace}\left(Y \rightarrow \nabla_{Y}^{P} X\right)$ of a vector field $X \in \mathcal{X}_{M}$ (defined equivalently in [10]) by

$$
\operatorname{div}_{P} X=-\nabla^{* P} X^{b}
$$

Indeed, at $x \in M$, we calculate

$$
\begin{aligned}
& \operatorname{div}_{P} X=\sum_{j}\left\langle\nabla_{e_{j}}^{P} X, e_{j}\right\rangle=\sum_{i, j}\left\langle P e_{j}, e_{i}\right\rangle\left\langle\nabla_{e_{i}} X, e_{j}\right\rangle \\
& =\sum_{i, j}\left\langle P e_{j}, e_{i}\right\rangle e_{i}\left\langle X, e_{j}\right\rangle=\sum_{i, j}\left\langle P e_{j}, e_{i}\right\rangle e_{i}\left(X^{b}\left(e_{j}\right)\right) \\
& =\sum_{i, j}\left\langle P e_{j}, e_{i}\right\rangle\left(\nabla_{e_{i}} X^{b}\right)\left(e_{j}\right)=\sum_{j}\left(\nabla_{P e_{j}} X^{b}\right)\left(e_{j}\right) \\
& =\sum_{j}\left(\nabla_{e_{j}}^{P} X^{b}\right)\left(e_{j}\right)=-\nabla^{* P} X^{b} .
\end{aligned}
$$

By (19) with $X^{b}=\omega$ and extended Divergence Theorem, see (17), we obtain (18).

Define the modified connection Laplacian by

$$
\bar{\Delta}^{P}=\nabla^{* P} \nabla^{P} .
$$

The next maximum principle generalizes ones used in the past.

Proposition 2. Let condition (15) hold for $P \in \operatorname{End}(T M)$ on a closed Riemannian manifold $(M, g)$ and let $S$ be a smooth tensor field such that $\left\langle\bar{\Delta}^{P} S, S\right\rangle \leq 0$. Then, $S$ is P-parallel.

Proof. We apply Formulas (18) and (20),

$$
0 \geq\left(\bar{\Delta}^{P} S, S\right)=\left(\nabla^{* P} \nabla^{P} S, S\right)=\left(\nabla^{P} S, \nabla^{P} S\right) \geq 0 ;
$$

hence, $\nabla^{P} S=0$. 
Example 2 (see [10]). We use popular geometrical structures that do not satisfy (1) to clarify the property (15). (a) An almost complex manifold $(M, J)$ (i.e., $J^{2}=-\mathrm{id}{ }_{T M}$ ) admits a Hermitian metric: $\langle J X, J Y\rangle=$ $\langle X, Y\rangle$ for $X, Y \in \mathfrak{X}_{M}$, e.g., [2]. If $P$ is an almost complex structure on $(M, g)$, then $P P^{*}=\mathrm{id}_{T M}$ and condition (15) obviously holds. This observation can be developed as follows.

(b) An $f$-structure (due to Yano, [22]) on a manifold $M$ is a non-null tensor $f \in \operatorname{End}(T M)$ of constant rank such that $f^{3}+f=0$. Such $f$ generalizes the almost complex and the almost contact structures. The restriction of $f$ to $\mathcal{D}=f(T M)$ determines a complex structure on it. An interesting case of $f$-structure on $M^{2 n+p}$ occurs when $\operatorname{ker} f$ is parallelizable for which there exist global vector fields $\xi_{i}, i \in\{1, \ldots, p\}$, with their dual 1-forms $\eta^{i}$, satisfying (see [23]) the following relations:

$$
f^{2}=-\mathrm{id}_{T M}+\sum_{i} \eta^{i} \otimes \xi_{i}, \quad \eta^{i}\left(\xi_{j}\right)=\delta_{j}^{i}
$$

It is known that $f \xi_{i}=0, \eta_{i} \circ f=0$ and $f$ has rank $2 n$. A Riemannian metric $g=\langle\cdot, \cdot\rangle$ is compatible, if $f^{*} f=\mathrm{id}_{T M}-\sum_{i} \eta^{i} \otimes \xi_{i}$. We have $f^{*}=-f$, and for $P=f$, we get

$$
\left(\operatorname{div} P P^{*}\right)(X)=-\sum_{j}\left\langle\nabla_{\xi_{j}} \xi_{j}+\left(\operatorname{div} \xi_{j}\right) \xi_{j}, X\right\rangle .
$$

Thus, (15) holds if and only if the distributions $f(T M)$ and $\operatorname{ker} f$ are both harmonic.

The metric $f$-structure for $p=1$ reduces to an almost contact metric structure, e.g., [2]. The condition (15) holds for an almost contact metric manifold $(M, \phi, \xi, \eta)$ and $P=\phi$ if and only if $\xi$ is a geodesic vector field $\left(\nabla_{\xi} \xi=0\right)$ and the distribution $\phi(T M)$ is harmonic $(\operatorname{div} \xi=0)$.

Definition 5. Define a skew-symmetric P-bracket $[\cdot, \cdot]_{P}: \mathcal{X}_{M} \times \mathcal{X}_{M} \rightarrow \mathcal{X}_{M}$ by

$$
[X, Y]_{P}=\nabla_{X}^{P} Y-\nabla_{Y}^{P} X
$$

Proposition 3. Condition $\mathfrak{D}^{P}=0$, see definition (6) with $\rho=P$, is equivalent to the following symmetry (on covariant components) of the $(1,2)$-tensor $\nabla^{P} P$ :

$$
\left(\nabla^{P} P\right)(X, Y)=\left(\nabla^{P} P\right)(Y, X), \quad X, Y \in \mathcal{X}_{M}
$$

Proof. Generally, we have

$$
\begin{aligned}
& \left(\nabla_{X}^{P} P\right)(Y)=\left(\nabla_{P X} P\right)(Y)=\nabla_{P X} P Y-P \nabla_{P X} Y \\
& {[P X, P Y]-P[X, Y]_{P}=\left(\nabla_{P X} P\right)(Y)-\left(\nabla_{P Y} P\right)(X) .}
\end{aligned}
$$

Thus,

$$
\mathfrak{D}^{P}(X, Y)=\left(\nabla_{X}^{P} P\right)(Y)-\left(\nabla_{Y}^{P} P\right)(X), \quad X, Y \in \mathcal{X}_{M},
$$

and the claim follows.

If condition (23) holds, then, by definitions (22) and (7), the $P$-derivative is torsion free; thus, $[\cdot, \cdot]_{P}$ defines a skew-symmetric algebroid structure with $E=T M$ and $\rho=P$.

Example 3. One can use structures of Example 2 to clarify the property (23).

(a) For an almost complex structure $P=J$ on TM, see Example 2(a), the property (23) describes a class of almost Hermitian manifolds which includes Kählerian manifolds, i.e., $\nabla J=0$. Differentiating $J^{2}=-\mathrm{id}_{T M}$, we obtain

$$
\left(\nabla_{X} J\right) J=-J\left(\nabla_{X} J\right), \quad X \in \mathfrak{X}_{M}
$$


Using (24), we can show that our class contains a wider class of nearly Kählerian manifolds, which are defined by $\left(\nabla_{X} J\right) X=0$, see [24]. Indeed, if $(M, J, g)$ is nearly Kählerian, then

$$
\begin{aligned}
0 & =\left(\nabla_{J(X+Y)} J\right) J(X+Y)=\left(\nabla_{J X} J\right) J X+\left(\nabla_{J X} J\right) J Y+\left(\nabla_{J Y} J\right) J X+\left(\nabla_{J Y} J\right) J Y \\
& =\left(\nabla_{J X} J\right) J Y+\left(\nabla_{J Y} J\right) J X=-J\left(\left(\nabla_{J X} J\right) Y+\left(\nabla_{J Y} J\right) X\right)
\end{aligned}
$$

from which (23) follows. There are many nearly Kählerian manifolds that are not Kählerian.

(b) The integrability tensor of a singular distribution $\mathcal{D}=P(T M)$ is defined by

$$
\mathcal{T}(X, Y)=\frac{1}{2} \tilde{P}\left(\nabla_{P X} P Y-\nabla_{P Y} P X\right)
$$

where $\tilde{P}: T M \rightarrow \mathcal{D}^{\perp}$ is the orthoprojector onto complementary orthogonal to $\mathcal{D}$ distribution, see [10]. Since $\tilde{P} \circ P=0$, we get $\mathcal{T}(X, Y)=\frac{1}{2} \tilde{P} \circ \mathfrak{D}^{P}(X, Y)$. Hence, (23) yields (but is not equivalent to) $\mathcal{T}=0$, in particular, for $P$ of constant rank, (23) yields integrability of $P(T M)$. Consequently, (23) is not satisfied for non-integrable regular distributions in Example 2(b). On the other hand, there exist integrable regular distributions (foliations) not satisfying condition (23). Indeed, taking a unit vector $Y \in \mathcal{D}^{\perp}$, since $P Y=0$, we obtain

$$
\mathfrak{D}^{P}(X, Y)=-P\left(\nabla_{P X} Y\right)=A_{Y}(P X),
$$

where $A_{Y}$ is the shape operator of $\mathcal{D}: A_{Y}(X)=-\left(\nabla_{X} Y\right)^{\top}$. For example, if $A_{Y}(X)=\lambda X$ (i.e., $\mathcal{D}$ is totally umbilical) and $\left.P\right|_{\mathcal{D}}$ is not identically zero, then $A_{Y}(P X) \neq 0$ for some $X$.

(c) For a metric $f$-structure on $M$, see Example 2(b), differentiating $(21)_{1}$, we get

$$
\begin{aligned}
& \left(\nabla_{X} f\right) f=-f\left(\nabla_{X} f\right)+\nabla_{X}\left(\eta^{i} \otimes \xi_{i}\right) \\
& \nabla_{X}\left(\eta^{i} \otimes \xi_{i}\right)(Y)=\left(\nabla_{X} \eta^{i}\right)(Y) \xi_{i}+\eta^{i}(Y) \nabla_{X} \xi_{i} .
\end{aligned}
$$

Consider a nearly Kähler f-structure on $M$, i.e., $\left(\nabla_{f X} f\right) f X=0$, see, e.g., [25]. Then,

$$
\begin{aligned}
& 0=\left(\nabla_{f(X+Y)} f\right) f(X+Y)=\left(\nabla_{f X} f\right) f X+\left(\nabla_{f X} f\right) f Y+\left(\nabla_{f Y} f\right) f X+\left(\nabla_{f Y} f\right) f Y \\
& =\left(\nabla_{f X} f\right) f Y+\left(\nabla_{f Y} f\right) f X=-f\left(\left(\nabla_{f X} f\right) Y+\left(\nabla_{f Y} f\right) X\right) \\
& -\left(\nabla_{f X} \eta^{i}\right)(Y) \xi_{i}-\left(\nabla_{f Y} \eta^{i}\right)(X) \xi_{i}-\eta^{i}(X) \nabla_{f Y} \xi_{i}-\eta^{i}(Y) \nabla_{f X} \xi_{i} .
\end{aligned}
$$

Taking $\mathcal{D}$-component of this, using $f^{*}=-f$ and assuming $\nabla \xi_{i}=0$ (in this case, $f(T M)$ is tangent to a totally geodesic foliation), we obtain (23). Thus, our class (23) contains nearly Kähler $f$-manifolds with parallel distribution $\operatorname{ker} f$. Remark that the class of nearly Kähler $f$-manifolds contains the class of Killing $f$-manifolds, i.e., $\left(\nabla_{X} f\right) X=0$, which is often defined by the condition that the fundamental form $F(X, Y)=\langle X, f Y\rangle$ is a Killing form.

For a metric $f$-structure with $p=1$, we have two appropriate cases. The class of almost contact metric manifolds $(M, g, \phi, \xi, \eta)$ with the condition (23) for $P=\phi$ includes:

- approximately cosymplectic manifolds (defined by $\left(\nabla_{X} \phi\right) X=0$ for $X \in \mathfrak{X}_{M}$, see [26]; such non-cosymplectic structure exists, e.g., on $\left.S^{7}\right)$ with parallel Reeb vector field, $\nabla \xi=0$.

- nearly Sasakian manifolds (defined by $\left(\nabla_{X} \phi\right) X=\langle X, X\rangle \xi-\eta(X) X$, see [2,27]) with parallel $\xi$. Unfortunately, any nearly Sasakian manifold of dimension greater than 5 is Sasakian.

\section{The Modified Hodge and Beltrami Laplacians}

First, we define the modified exterior derivative and its $L^{2}$-adjoint.

The P-exterior derivative of $\omega \in \Lambda^{k}(T M)$ as a $(k+1)$-form

$$
d^{P} \omega\left(X_{0}, \ldots, X_{k}\right)=\sum_{i}(-1)^{i}\left(\nabla_{X_{i}}^{P} \omega\right)\left(X_{0}, \ldots, \widehat{X}_{i}, \ldots X_{k}\right)
$$


For a differential form $\omega_{p}$, the form $\nabla^{P} \omega$ given in (13) is not skew-symmetric, but the form $d^{P} \omega$ is skew-symmetric. For a function $f$ on $M$, we have $d^{P} f=\nabla^{P} f$, see Definition 4. Put $\delta^{P}=\nabla^{* P}$ : $\Lambda^{k+1}(T M) \rightarrow \Lambda^{k}(T M)$ for differential forms. By (14), we have

$$
\left(\delta^{P} \omega\right)\left(X_{2}, \ldots, X_{k}\right)=-\sum_{i}\left(\nabla_{e_{i}}^{P} \omega\right)\left(e_{i}, X_{2}, \ldots, X_{k}\right)
$$

Similarly to Proposition 1, we have the following.

Proposition 4. If condition (15) holds on a closed $(M, g)$ then $\delta^{P}$ is $L^{2}$-adjoint to $d^{P}$, i.e.,

$$
\left(\delta^{P} \omega_{k+1}, \omega_{k}\right)=\left(\omega_{k+1}, d^{P} \omega_{k}\right),
$$

for any two differential forms $\omega_{k} \in \Lambda^{k}(T M)$ and $\omega_{k+1} \in \Lambda^{k+1}(T M)$.

Proof. We use $\left(\nabla^{P} \omega_{k}, \omega_{k+1}\right)=\left(\omega_{k}, \delta^{P} \omega_{k+1}\right)$, which requires (15), and

$$
\begin{aligned}
& \left\langle d^{P} \omega_{k}, \omega_{k+1}\right\rangle=\sum_{u=0}^{k}(-1)^{i} \nabla_{\partial_{i_{u}}}^{P} \omega_{k}\left(\partial_{i_{1}}, \ldots, \widehat{\partial}_{i_{u}}, \ldots, \partial_{i_{k}}\right) g^{i_{0} j_{0}} \ldots g^{i_{k} j_{k}} \omega_{k+1}\left(\partial_{i_{1}}, \ldots, \partial_{i_{k}}\right) \\
& =\left(\nabla_{\partial_{i_{0}}}^{P} \omega_{k}\left(\partial_{i_{1}}, \ldots, \partial_{i_{k}}\right)\right) g^{i_{0} j_{0}} \ldots g^{i_{k} j_{k}} \omega_{k+1}\left(\partial_{j_{0}}, \ldots, \partial_{j_{k}}\right)=\left\langle\nabla^{P} \omega_{k}, \omega_{k+1}\right\rangle,
\end{aligned}
$$

as in the classical case. Thus, $\left(d^{P} \omega_{k}, \omega_{k+1}\right)=\left(\nabla^{P} \omega_{k}, \omega_{k+1}\right)$. Using condition (15), by (18), we get $\left(\nabla^{P} \omega_{k}, \omega_{k+1}\right)=\left(\omega_{k}, \nabla^{P *} \omega_{k+1}\right)=\left(\omega_{k+1}, d^{P} \omega_{k}\right)$.

Proposition 5. The $d^{P}: \Lambda^{k}(T M) \rightarrow \Lambda^{k+1}(T M)(1 \leq k \leq n)$ is an exterior derivation, see the definition in Section 2, that is,

$$
\begin{aligned}
d^{P} \omega\left(X_{0}, \ldots, X_{k}\right) & =\sum_{i=0}^{k}(-1)^{i} P X_{i}\left(\omega\left(X_{0}, \ldots, \widehat{X}_{i}, \ldots, X_{k}\right)\right) \\
& +\sum_{0 \leq i<j \leq k}(-1)^{i+j} \omega\left([X, Y]_{P}, X_{0}, \ldots, \widehat{X}_{i}, \ldots, \widehat{X}_{j}, \ldots, X_{k}\right) .
\end{aligned}
$$

Proof. We have

$$
\begin{aligned}
& d^{P} \omega\left(X_{0}, \ldots, X_{k}\right)=\sum_{i=0}^{k}(-1)^{i}\left(\nabla_{P X_{i}} \omega\right)\left(X_{0}, \ldots, \widehat{X}_{i}, \ldots, X_{k}\right) \\
& =\sum_{i=0}^{k}(-1)^{i} P X_{i}\left(\omega\left(X_{0}, \ldots, \widehat{X}_{i}, \ldots, X_{k}\right)\right) \\
& +\sum_{i=0}^{k}(-1)^{i}\left(\sum_{j=0}^{i-1} \omega\left(X_{0}, \ldots, \nabla_{P X_{i}} X_{j}, \ldots, \widehat{X}_{i}, \ldots, X_{k}\right)\right. \\
& \left.+\sum_{j=i+1}^{k} \omega\left(X_{0}, \ldots, \widehat{X}_{i}, \ldots, \nabla_{P X_{i}} X_{j}, \ldots, X_{k}\right)\right) \\
& =\sum_{i=0}^{k}(-1)^{i} P X_{i}\left(\omega\left(X_{0}, \ldots, \widehat{X}_{i}, \ldots, X_{k}\right)\right) \\
& +\sum_{0 \leq i<j \leq k}(-1)^{i+j} \omega\left(\nabla_{P X_{i}} X_{j}-\nabla_{P X_{j}} X_{i}, X_{0}, \ldots, \widehat{X}_{i}, \ldots, \widehat{X}_{j}, \ldots, X_{k}\right) .
\end{aligned}
$$

The use of (12) and (22) completes the proof.

Next, we extend the definition of the Hodge Laplacian $\Delta_{H}=d \delta+\delta d: \Lambda^{k}(T M) \rightarrow \Lambda^{k}(T M)$.

Definition 6. Define the Hodge type Laplacian $\Delta_{H}^{P}$ for differential forms by

$$
\Delta_{H}^{P}=d^{P} \delta^{P}+\delta^{P} d^{P} .
$$

We say that a differential form $\omega$ is $P$-harmonic if $\Delta_{H}^{P} \omega=0$. 
Remark 1. The P-harmonic forms have similar properties as in the classical case, see, e.g., ([15], Lemma 9.1.1). Let condition (15) hold on a closed $(M, g)$. For $\omega \in \Lambda^{k}(T M)$, using Proposition 4 and (25), we have

$$
\left(\Delta_{H}^{P} \omega, \omega\right)=\left(d^{P} \omega, d^{P} \omega\right)+\left(\delta^{P} \omega, \delta^{P} \omega\right),
$$

thus, a differential form $\omega$ is P-harmonic if and only if

$$
d^{P} \omega=0, \quad \delta^{P} \omega=0 .
$$

Observe that, if $\Delta_{H}^{P} \omega=0$ and $\omega=d^{P} \theta$, then $\delta^{P} d^{P} \theta=\delta^{P} \omega=0$.

It follows that

$$
(\omega, \omega)=\left(d^{P} \theta, d^{P} \theta\right)=\left(\theta, \delta^{P} d^{P} \theta\right)=\left(\theta, \delta^{P} \omega\right)=0 .
$$

Thus, if $\omega$ is P-harmonic and $\omega=d^{P} \theta$, then $\omega=0$.

For functions, $\Delta_{B}^{P} f=\operatorname{div}^{P}\left(\nabla^{P} f\right)$ is the Beltrami type Laplacian. For any $x \in M$, we have

$$
\Delta_{B}^{P} f(x)=\sum_{i}\left(P e_{i}\right)^{2}(f)(x),
$$

where $\left(e_{i}\right)$ is an orthonormal frame such that $\nabla_{Y} e_{i}=0$ for all $Y \in T_{X} M$. A function $f \in C^{\infty}(M)$ is called $P$-subharmonic if $\Delta_{B}^{P} f \leq 0$. If $\Delta_{B}^{P} f \geq 0$, then $f$ is called P-superharmonic.

Lemma 2 (see Proposition 2.8 in [10]). Let $(M, g)$ be a complete open Riemannian manifold endowed with a vector field $X$ such that $\operatorname{div}_{P} X \geq 0\left(\right.$ or $\left.\operatorname{div}_{P} X \leq 0\right)$, where $P \in \operatorname{End}(T M)$ such that (15) and $\left\|P P^{*}(X)\right\|_{g} \in \mathrm{L}^{1}(M, g)$ hold. Then, $\operatorname{div}_{P} X \equiv 0$.

Consider the following system of singular distributions: $\mathcal{D}_{1}=\mathcal{D}, \mathcal{D}_{2}=\mathcal{D}_{1}+\left[\mathcal{D}, \mathcal{D}_{1}\right]$, etc. The distribution $\mathcal{D}$ is said to be bracket-generating of the step $r \in \mathbb{N}$ if $\mathcal{D}_{r}=T M$, see, e.g., [4]. Note that integrable distributions, i.e., $[X, Y] \in \mathfrak{X}_{\mathcal{D}}\left(X, Y \in \mathfrak{X}_{\mathcal{D}}\right)$, are not bracket-generating. The condition $\nabla^{P} f=0$ means that $f$ is constant along the (integral curves of) $P(T M)$; moreover, $f=$ const on $M$ when $\mathcal{D}=P(T M)$ is bracket-generating.

The next theorem for $P=\mathrm{id}_{T M}$ reduces to the classical result on subharmonic functions.

Theorem 1. Let condition (15) hold and let $f \in C^{2}(M)$ satisfy $\Delta_{B}^{P} f \geq 0$ or $\Delta_{B}^{P} f \leq 0$. Suppose that any of the following conditions hold: a) $(M, g)$ is closed; $b)(M, g)$ is open complete, $\left\|P P^{*} \nabla^{P} f\right\| \in L^{1}(M, g)$ and $\left\|f P P^{*} \nabla^{P} f\right\| \in L^{1}(M, g)$. Then, $\nabla^{P} f=0$; moreover, if $P(T M)$ is bracket-generating, then $f=$ const.

Proof. Set $X=\nabla^{P} f$, then $\Delta_{B}^{P} f=\operatorname{div}_{P} X$. a) Using the extended Divergence Theorem, see (17), we get $\Delta_{B}^{P} f \equiv 0$. Using the equality

$$
\operatorname{div}_{P}(f \cdot Y)=f \cdot \operatorname{div}_{P} Y+\left\langle\nabla^{P} f, Y\right\rangle
$$

with $Y=\nabla^{P} f$ and again the extended Divergence Theorem with $X=f \nabla^{P} f$, we get $\left(\nabla^{P} f, \nabla^{P} f\right)=0$, hence $\nabla^{P} f=0$. b) By Lemma 2 with $X=\nabla^{P} f$ and condition $\left\|P P^{*} \nabla^{P} f\right\| \in L^{1}(M, g)$, we get $\Delta_{B}^{P} f \equiv 0$. Using the Formula (27), with $Y=\nabla^{P} f$, Lemma 2 with $X=f \nabla^{P} f$ and condition $\left\|f P P^{*} \nabla^{P} f\right\| \in$ $L^{1}(M, g)$, we get $\left(\nabla^{P} f, \nabla^{P} f\right)=0$, hence $\nabla^{P} f=0$. If the distribution $P(T M)$ is bracket-generating, then using Chow's theorem [28] completes the proof for both cases.

\section{The Modified Curvature Tensor}

We will use $\nabla^{P}$ to construct the second $P$-derivative on tensors and then the $P$-curvature. 
Definition 7. Define the second P-derivative of an $(s, k)$-tensor $S$ by

$$
\left(\nabla^{P}\right)_{X, Y}^{2} S=\nabla_{X}^{P}\left(\nabla_{Y}^{P} S\right)-\nabla_{\nabla_{X}^{P} Y}^{P} S .
$$

Define the P-curvature of connection $\nabla^{P}$ by

$$
R_{X, Y}^{P} Z=\left(\nabla^{P}\right)_{X, Y}^{2} Z-\left(\nabla^{P}\right)_{Y, X}^{2} Z=\nabla_{X}^{P} \nabla_{Y}^{\rho} Z-\nabla_{Y}^{P} \nabla_{X}^{P} Z-\nabla_{[X, Y]_{P}}^{P} Z .
$$

See (8) with $\rho=P$, and set

$$
R^{P}(X, Y, Z, W)=\left\langle R_{X, Y}^{P} Z, W\right\rangle, \quad X, Y, Z, W \in \mathcal{X}_{M}
$$

The P-Ricci tensor is defined by the standard way: $\operatorname{Ric}^{P}(X, Y)=\sum_{i} R^{P}\left(X, e_{i}, e_{i}, Y\right)$.

Set $\mathcal{J}_{P}(X, Y, Z)=\sum_{\text {circ. }}\left[X,[Y, Z]_{P}\right]_{P}$, see (5). By (9) and since $\nabla^{P}$ is torsionless, we get

$$
\sum_{\text {circ. }} R_{X, Y}^{P} Z=\mathcal{J}_{P}(X, Y, Z) .
$$

The $R^{P}$ acts on tensor fields as follows:

- $R_{X, Y}^{P}(f)=\mathfrak{D}^{P}(X, Y)(f)$ on functions $f \in C^{\infty}(M)$,

- $R_{X, Y}^{P} Z$ given by Formula (28) on vector fields $Z \in \mathcal{X}_{M}$,

- $\left(R_{X, Y}^{P} \omega\right)(Z)=\mathfrak{D}^{P}(X, Y)(\omega(Z))-\omega\left(R_{X, Y}^{P} Z\right)$ on differential 1-forms,

- $\quad$ and similarly on $(0, k)$-tensors:

$$
\left(R_{X, Y}^{P} S\right)\left(X_{1}, \ldots, X_{k}\right)=\mathfrak{D}^{P}(X, Y)\left(S\left(X_{1}, \ldots, X_{k}\right)\right)-\sum_{i} S\left(X_{1}, \ldots R_{X, Y}^{P} X_{i}, \ldots, X_{k}\right) .
$$

By the above calculations, the action of $R_{X, Y}^{P}$ on tensors is $C^{\infty}(M)$-linear if $\mathfrak{D}^{P}=0$, i.e., (23) holds; in this case, we conclude the same about the $C^{\infty}(M)$-homogeneity of $R_{X, Y}^{P} \omega$ in $\omega$. In other words, the action of the endomorphism $R_{X, Y}^{P}$ on differential forms is defined as in the classical case (see [15], Section 2.3.1): as a one degree derivation acting trivially on functions, as an endomorphism on vector fields and as an adjoint endomorphism on forms.

Based on the above arguments, we assume (15) and (23) in the rest of the article.

We will show that the $P$-curvature has some symmetry properties, similar to the classical variants, when $P=\mathrm{id} T M$ and according to ([15], Section 3.1.1).

Proposition 6. For $X, Y, Z, W \in \mathcal{X}_{M}, \omega \in \Lambda^{1}(T M)$ and $f \in C^{\infty}(M)$, we have

1. $R_{X, Y}^{P} f=0 ; \quad R_{X, Y}^{P} g=0$;

2. $R_{X, Y}^{P} Z=R_{P X, P Y} Z ; \quad\left(R_{X, Y}^{P} \omega\right)(Z)=-\omega\left(R_{X, Y}^{P} Z\right)$;

3. $R_{X, Y}^{P} T=R_{P X, P Y} T$ for every tensor $T$;

4. $P R_{X, Y}^{P} Z=R_{X, Y}^{P} P Z$;

5. $\quad R^{P}\left(X, Y, Z,\left(P^{*} P\right) W\right)=R^{P}(X, Y, P Z, P W)=R(P X, P Y, P Z, P W)$;

6. $R^{P}(X, Y, Z, W)=-R^{P}(Y, X, Z, W)=-R^{P}(X, Y, W, Z)$;

7. $\left\langle R_{X, Y}^{P} Z, W\right\rangle=-\left\langle R_{X, Y}^{P} W, Z\right\rangle$. 
The endomorphism $P$ of $T M$ induces endomorphisms of $\Lambda^{2}(T M): \mathcal{P}$ and its adjoint $\mathcal{P}^{*}$,

$$
\mathcal{P}(X \wedge Y)=P X \wedge P Y, \quad \mathcal{P}^{*}(X \wedge Y)=P^{*} X \wedge P^{*} Y .
$$

Indeed,

$$
\begin{aligned}
\langle\mathcal{P}(X \wedge Y), Z \wedge W\rangle & =\left|\begin{array}{cc}
\langle P X, Z\rangle & \langle P X, W\rangle \\
\langle P Y, Z\rangle & \langle P Y, W\rangle
\end{array}\right| \\
& =\left|\begin{array}{cc}
\left\langle X, P^{*} Z\right\rangle & \left\langle X, P^{*} W\right\rangle \\
\left\langle Y, P^{*} Z\right\rangle & \left\langle Y, P^{*} W\right\rangle
\end{array}\right|=\left\langle X \wedge Y, \mathcal{P}^{*}(Z \wedge W)\right\rangle .
\end{aligned}
$$

The curvature tensor $R_{X, Y}$ can be seen as a self-adjoint linear operator $\mathcal{R}$ on the space $\Lambda^{2}(T M)$ of bivectors, called the curvature operator (note the reversal of $Z$ and $W$ ):

$$
\langle\mathcal{R}(X \wedge Y), Z \wedge W\rangle=R(X, Y, W, Z), \quad \mathcal{R}^{*}=\mathcal{R} .
$$

Similarly, consider $R_{X, Y}^{P}=R_{P X, P Y}$ as a linear operator $\mathcal{R}^{P}=\mathcal{R} \circ \mathcal{P}$ or as a corresponding bilinear form on $\Lambda^{2}(T M)$, that is,

$$
\begin{aligned}
& \mathcal{R}^{P}(X \wedge Y)=\mathcal{R} \circ \mathcal{P}(X \wedge Y)=\mathcal{R}(P X \wedge P Y), \\
& \mathcal{R}^{P}(X \wedge Y, Z \wedge W)=\left\langle\mathcal{R}^{P}(X \wedge Y), Z \wedge W\right\rangle .
\end{aligned}
$$

Using known properties of $\mathcal{R}$ and $R^{P}$, we have

$$
\left\langle\mathcal{R}^{P}(X \wedge Y), Z \wedge W\right\rangle=\langle\mathcal{R}(P X \wedge P Y), Z \wedge W\rangle=R^{P}(X, Y, W, Z) .
$$

Remark 2. The $\mathcal{R}^{P}$ on $\Lambda^{2}(T M)$ generally is not self-adjoint:

$$
\left(\mathcal{R}^{P}\right)^{*}=(\mathcal{R} \circ \mathcal{P})^{*}=\mathcal{P}^{*} \circ \mathcal{R} \neq \mathcal{R} \circ \mathcal{P}=\mathcal{R}^{P} .
$$

\section{The Weitzenböck Type Curvature Operator}

The next definition generalizes the Weitzenböck curvature operator (3).

Definition 8. Define the P-Weitzenböck curvature operator on $(0, k)$-tensors $S$ over $(M, g)$ by

$$
\begin{aligned}
\mathcal{R i c}^{P}(S)\left(X_{1}, \ldots, X_{k}\right)=\sum_{\alpha=1}^{k} \sum_{i=1}^{n}\left(R_{e_{i}, X_{\alpha}}^{P} S\right)(\underbrace{X_{1}, \ldots, e_{i}}_{a}, \ldots, X_{k}) \\
=-2 \sum_{i, m, a ; b<a} R^{P}\left(e_{i}, X_{a}, e_{m}, X_{b}\right) \cdot S(\underbrace{X_{1}, \ldots, e_{m}}_{b}, \underbrace{\ldots, e_{i}}_{a-b}, \ldots, X_{k}) \\
\quad+\sum_{i, a} \operatorname{Ric}^{P}\left(e_{i}, X_{a}\right) \cdot S(\underbrace{X_{1}, \ldots, e_{i}}_{a}, \ldots, X_{k}),
\end{aligned}
$$

or, in coordinates, $\mathcal{R i c}^{P}(S)_{i_{1}, \ldots, i_{k}}=-2 \sum_{a<b} R_{j i_{a} p i_{b}}^{P} S_{i_{1} \ldots \ldots \ldots i_{k}}^{j}+\sum_{a} \operatorname{Ric}_{i_{a j} j}^{P} S_{i_{1} \ldots \ldots i_{k}}{ }^{j}$

For a differential form $\omega$, the $\mathcal{R i c}^{P}(\omega)$ is skew-symmetric. For vector fields and 1-forms, the $\mathcal{R i c}^{P}$ is similar to the classical Ricci tensor.

The following result extends a Weitzenböck theorem (see [15], Theorem 9.4.1).

Theorem 2. The following Weitzenböck type decomposition formula holds:

$$
\Delta_{H}^{P}=\bar{\Delta}^{P}+\mathcal{R i c}^{P} .
$$


Proof. We follow the same lines as in the proof of ([15], Theorem 9.4.1): for $\omega \in \Lambda^{k}(T M)$,

$$
\begin{aligned}
& d^{P} \delta^{P} \omega\left(X_{1}, \ldots, X_{k}\right)=-\sum_{i, \alpha}(-1)^{\alpha+1}\left(\nabla_{X_{\alpha}, e_{i}}^{P, 2} \omega\right)(e_{i}, \underbrace{X_{1}, \ldots, \widehat{X_{\alpha}}}_{i}, \ldots, X_{k}) \\
& =-\sum_{i, \alpha}\left(\nabla_{X_{\alpha}, e_{i}}^{P, 2} \omega\right)(\underbrace{X_{1}, \ldots, e_{i}}_{\alpha}, \ldots, X_{k}), \\
& \delta^{P} d^{P} \omega\left(X_{1}, \ldots, X_{k}\right)=-\sum_{i}\left(\nabla_{e_{i}, e_{i}}^{P, 2} \omega\right)\left(X_{1}, \ldots, X_{k}\right) \\
& -\sum_{i, \alpha}(-1)^{\alpha}\left(\nabla_{e_{i}, X_{\alpha}}^{P, 2} \omega\right)(e_{i}, \underbrace{X_{1} \ldots, \widehat{X}_{\alpha}}_{i}, \ldots, X_{k}) \\
& =\left(\nabla^{* P} \nabla^{P} \omega\right)\left(X_{1}, \ldots, X_{k}\right)+\sum_{i, \alpha}\left(\nabla_{e_{i}, X_{\alpha}}^{P, 2} \omega\right)(\underbrace{X_{1} \ldots, e_{i}}_{\alpha}, \ldots, X_{k}),
\end{aligned}
$$

where $1 \leq i \leq n$ and $1 \leq \alpha \leq k$. Substituting the above into (25) yields (31).

From $\Delta_{H} S=\bar{\Delta} S+\mathcal{R} i c(S)$, see (2), we obtain the Bochner-Weitzenböck formula

$$
\frac{1}{2} \Delta_{B}\left(\|S\|^{2}\right)=-\left\langle\Delta_{H} S, S\right\rangle+\|\nabla S\|^{2}+\langle\mathcal{R i c}(S), S\rangle,
$$

where $\Delta_{B}=$ div o grad is the Beltrami Laplacian on $C^{\infty}(M)$, e.g., [15-18]. In the next proposition, we generalize (32) for distributions.

Proposition 7. The modified Bochner-Weitzenböck formula is valid:

$$
\frac{1}{2} \Delta_{B}^{P}\left(\|S\|^{2}\right)=-\left\langle\Delta_{H}^{P} S, S\right\rangle+\left\|\nabla^{P} S\right\|^{2}+\left\langle\mathcal{R i c}^{P}(S), S\right\rangle .
$$

Proof. First, we find

$$
\frac{1}{2} \Delta_{B}^{P}\left(\|S\|^{2}\right)=-\left\langle\bar{\Delta}^{P} S, S\right\rangle+\left\|\nabla^{P} S\right\|^{2}
$$

Indeed, take an orthonormal frame $\left(e_{i}\right)$ such that $\nabla_{Y} e_{i}=0$ for all $Y \in T_{X} M$ and, in order to simplify calculations, assume that $s=t=1$. By (26), and taking into account the properties of normal coordinates, we have at $x \in M$,

$$
\begin{aligned}
\frac{1}{2} \Delta_{B}^{P}\left(\|S\|^{2}\right) & =\frac{1}{2} \sum_{i}\left(P e_{i}\right)^{2}\langle S, S\rangle=\sum_{i}\left(P e_{i}\right)\left\langle\nabla_{P e_{i}} S, S\right\rangle \\
& =\sum_{i}\left[\left\langle\nabla_{P e_{i}} \nabla_{P e_{i}} S, S\right\rangle+\left\langle\nabla_{P e_{i}} S, \nabla_{P e_{i}} S\right\rangle\right] \\
& =-\left\langle\bar{\Delta}^{P} S, S\right\rangle+\left\|\nabla^{P} S\right\|^{2}
\end{aligned}
$$

Then, using (31), we get (33).

\section{Applications of the Weitzenböck Type Curvature Operator}

Here, rewrite the $P$-Weitzenböck curvature operator using an orthonormal basis $\left(\xi_{a}\right)$ of skew-symmetric transformations $\mathfrak{s o}(T M)$ and give some applications of $\mathcal{R i c}^{P}$.

For every bivector $X \wedge Y \in \Lambda^{2}(T M)$, we build a map $\mathcal{R}^{P}(X \wedge Y): \mathcal{X}_{M} \rightarrow \mathcal{X}_{M}$, given by

$$
\left\langle\mathcal{R}^{P}(X \wedge Y) Z, W\right\rangle=\left\langle\mathcal{R}^{P}(X \wedge Y), W \wedge Z\right\rangle=R(P X, P Y, Z, W) .
$$

Since bivectors are generators of the vector space $\Lambda^{2}(T M)$, we obtain in this way a map $\mathcal{R}^{P}(\xi)$ : $\mathcal{X}_{M} \rightarrow \mathcal{X}_{M}$ (similarly to algebraic curvature operator $\mathcal{R}(\xi)$ ). 
Lemma 3. The map $\mathcal{R}^{P}(\xi)$, where $\xi \in \Lambda^{2}(T M)$, is skew-symmetric: $\left\langle\mathcal{R}^{P}(\xi) W, Z\right\rangle=-\left\langle\mathcal{R}^{P}(\xi) Z, W\right\rangle$.

Proof. It suffices to check the statement for the generators. We have, using Proposition 6,

$$
\left\langle\mathcal{R}^{P}(X \wedge Y) Z, W\right\rangle=R(P X, P Y, Z, W)=-R(P X, P Y, W, Z)=-\left\langle\mathcal{R}^{P}(X \wedge Y) W, Z\right\rangle .
$$

Thus, the statement follows.

The associated $P$-curvature operator is given by $\left\langle\mathcal{R}^{P}(X \wedge Y), Z \wedge W\right\rangle=R(P X, P Y, W, Z)$.

We are based on the fact that, if $X$ and $Y$ are orthonormal, then $X \wedge Y$ is a unit bivector, while the corresponding skew-symmetric operator (a counterclockwise rotation of $\pi / 2$ in the plane $\operatorname{span}(X, Y)$ ) has Euclidean norm $\sqrt{2}$. To simplify calculations, we assume that $\mathfrak{s o}(T M)$ is endowed with metric induced from $\Lambda^{2}(T M)$, see, e.g., [19]. If $L \in \mathfrak{s o}(T M)$, then

$$
(L S)\left(X_{1}, \ldots, X_{k}\right)=-\sum_{i} S\left(X_{1}, \ldots, L\left(X_{i}\right), \ldots, X_{k}\right) .
$$

Let $\left\{\xi_{a}\right\}$ be an orthonormal base of skew-symmetric transformations such that $\left(\xi_{a}\right)_{x} \in \mathfrak{s o}\left(T_{x} M\right)$ for $x$ in an open set $U \subset M$. By (34), for any $(0, k)$-tensor $S$,

$$
\left(\xi_{\alpha} S\right)\left(X_{1}, \ldots, X_{k}\right)=-\sum_{i} S\left(X_{1}, \ldots, \xi_{\alpha}\left(X_{i}\right), \ldots, X_{k}\right) ;
$$

The $\mathcal{R}^{P}(X \wedge Y)$ on $\Lambda^{2}(T M)$ can be decomposed using $\left\{\xi_{a}\right\}$.

Lemma 4. We have

$$
\mathcal{R}^{P}(X \wedge Y)=-\sum_{\alpha}\left\langle\mathcal{P}^{*} \circ \mathcal{R}\left(\xi_{\alpha}\right) X, Y\right\rangle \xi_{\alpha}=-\sum_{\alpha}\left\langle\mathcal{R}\left(\xi_{\alpha}\right) P X, P Y\right\rangle \xi_{\alpha} .
$$

Proof. Using that $\left(\mathcal{R}^{P}\right)^{*}=\mathcal{P}^{*} \circ \mathcal{R}$ and Lemma 3, we have:

$$
\begin{aligned}
\mathcal{R}^{P}(X \wedge Y) & =\sum_{\alpha}\left\langle\mathcal{R}^{P}(X \wedge Y), \xi_{\alpha}\right\rangle \xi_{\alpha} \\
& =\sum_{\alpha}\left\langle\mathcal{P}^{*} \circ \mathcal{R}\left(\xi_{\alpha}\right), X \wedge Y\right\rangle \xi_{\alpha}=-\sum_{\alpha}\left\langle\mathcal{R}\left(\xi_{\alpha}\right) P X, P Y\right\rangle \xi_{\alpha} .
\end{aligned}
$$

Lemma 4 allows us to rewrite the Weitzenböck type curvature operator (30).

Proposition 8. If $S$ is a $(0, k)$-tensor on $(M, g)$, then

$$
\mathcal{R i c}^{P}(S)=-\sum_{\alpha} \mathcal{R}^{P}\left(\xi_{a}\right)\left(\xi_{a} S\right), \quad\left(\mathcal{R i c}^{P}(S)\right)^{*}=\mathcal{R i c}^{P^{*}}(S) .
$$

In particular, if $P$ is self-adjoint, then $\operatorname{Ric}^{P}$ is self-adjoint too.

Proof. We follow similar arguments as in the proof of ([15], Lemma 9.3.3):

$$
\begin{aligned}
\mathcal{R i c}^{P}(S)\left(X_{1}, \ldots, X_{k}\right) & =\sum_{i, j}\left(\mathcal{R}^{P}\left(e_{j} \wedge X_{i}\right) S\right)(\underbrace{X_{1}, \ldots, e_{j}}_{i}, \ldots, X_{k}) \\
& =-\sum_{i, j, \alpha}\left\langle\mathcal{P}^{*} \circ \mathcal{R}\left(\xi_{\alpha}\right) e_{j}, X_{i}\right\rangle\left(\xi_{\alpha} S\right)\left(X_{1}, \ldots, e_{j}, \ldots, X_{k}\right) \\
& =-\sum_{i, j, \alpha}\left(\xi_{\alpha} S\right)\left(X_{1}, \ldots,\left\langle\mathcal{P}^{*} \circ \mathcal{R}\left(\xi_{\alpha}\right) e_{j}, X_{i}\right\rangle e_{j}, \ldots, X_{k}\right) \\
& =-\sum_{i, j, \alpha}\left(\xi_{\alpha} S\right)\left(X_{1}, \ldots,\left\langle e_{j}, \mathcal{R}^{P}\left(\xi_{\alpha}\right) X_{i}\right\rangle e_{j}, \ldots, X_{k}\right) \\
& =-\sum_{i, \alpha}\left(\xi_{\alpha} S\right)\left(X_{1}, \ldots, \mathcal{R}^{P}\left(\xi_{\alpha}\right) X_{i}, \ldots, X_{k}\right) \\
& =-\sum_{\alpha}\left(\mathcal{R}^{P}\left(\xi_{\alpha}\right)\left(\xi_{\alpha} S\right)\right)\left(X_{1}, \ldots, X_{k}\right) .
\end{aligned}
$$


Thus, the first claim follows. Since $\mathcal{R}: \Lambda^{2}(T M) \rightarrow \Lambda^{2}(T M)$ is self-adjoint, there is a local orthonormal base $\left\{\xi_{a}\right\}$ of $\Lambda^{2}(T M)$ such that $\mathcal{R}\left(\xi_{a}\right)=\lambda_{a} \xi_{a}$. Using this base, for any $(0, k)$-tensors $S_{1}$ and $S_{2}$, we have

$$
\begin{aligned}
\left\langle\mathcal{R i c}^{P}\left(S_{2}\right), S_{1}\right\rangle & =-\sum_{\alpha}\left\langle\mathcal{R}^{P}\left(\xi_{\alpha}\right)\left(\xi_{\alpha} S_{2}\right), S_{1}\right\rangle=-\sum_{\alpha}\left\langle\xi_{\alpha} S_{2},\left(\mathcal{R}^{P}\right)^{*}\left(\xi_{\alpha}\right) S_{1}\right\rangle \\
& =\sum_{\alpha}\left\langle\xi_{\alpha} S_{2}, \mathcal{P}^{*} \circ \mathcal{R}\left(\xi_{\alpha}\right)\left(S_{1}\right)\right\rangle=\sum_{\alpha} \lambda_{\alpha}\left\langle\mathcal{P}\left(\xi_{\alpha} S_{2}\right), \xi_{\alpha} S_{1}\right\rangle,
\end{aligned}
$$

and, similarly,

$$
\left\langle S_{2}, \mathcal{R i c}^{P^{*}}\left(S_{1}\right)\right\rangle=\sum_{\alpha} \lambda_{\alpha}\left\langle\xi_{\alpha} S_{2}, \mathcal{P}^{*}\left(\xi_{\alpha} S_{1}\right)\right\rangle=\sum_{\alpha} \lambda_{\alpha}\left\langle\mathcal{P}\left(\xi_{\alpha} S_{2}\right), \xi_{\alpha} S_{1}\right\rangle .
$$

Thus, the second claim follows.

For a $(0, k)$-tensor $S$ on $M$, define a $(0, k)$-tensor $\widehat{S}$ with values in $\Lambda^{2}(T M)$ implicitly by

$$
\left\langle L, \widehat{S}\left(X_{1}, \ldots, X_{k}\right)\right\rangle=(L S)\left(X_{1}, \ldots, X_{k}\right), \quad L \in \mathfrak{s o}(T M),
$$

see [19]. The following result generalizes ([19], Lemma 3.5).

Proposition 9. If $S_{1}, S_{2}$ are $(0, k)$-tensors, then

$$
\left\langle\mathcal{R i c}^{P}\left(S_{1}\right), S_{2}\right\rangle=\left\langle\widehat{S}_{1}, \mathcal{R}^{P}\left(\widehat{S}_{2}\right)\right\rangle .
$$

Proof. Using a local orthonormal base $\left\{\tilde{\xi}_{\alpha}\right\}$ of $\Lambda^{2}(T M)$, we get

$$
\begin{aligned}
\left\langle\mathcal{R i c}^{P}\left(S_{1}\right), S_{2}\right\rangle & =\sum_{\alpha}\left\langle\mathcal{R}^{P}\left(\xi_{\alpha}\right)\left(\xi_{\alpha} S_{1}\right), S_{2}\right\rangle=\sum_{\alpha}\left\langle\xi_{\alpha} S_{1}, \mathcal{R}^{P *}\left(\xi_{\alpha}\right) S_{2}\right\rangle \\
& =\sum_{\alpha, i}\left(\xi_{\alpha} S_{1}\right)\left(e_{i_{1}}, \ldots, e_{i_{k}}\right)\left(\mathcal{R}^{P *}\left(\xi_{\alpha}\right) S_{2}\right)\left(e_{i_{1}}, \ldots, e_{i_{k}}\right) \\
& =\sum_{\alpha, i}\left\langle\xi_{\alpha}, \widehat{S}_{1}\left(e_{i_{1}}, \ldots, e_{i_{k}}\right)\right\rangle\left\langle\mathcal{R}^{P *}\left(\xi_{\alpha}\right), \widehat{S}_{2}\left(e_{i_{1}}, \ldots, e_{i_{k}}\right)\right\rangle \\
& =\sum_{\alpha, i}\left\langle\mathcal{R}^{P *}\left(\left\langle\xi_{\alpha}, \widehat{S}_{1}\left(e_{i_{1}}, \ldots, e_{i_{k}}\right)\right\rangle \xi_{\alpha}\right), \widehat{S}_{2}\left(e_{i_{1}}, \ldots, e_{i_{k}}\right)\right\rangle \\
& =\sum_{i}\left\langle\mathcal{R}^{P *}\left(\widehat{S}_{1}\left(e_{i_{1}}, \ldots, e_{i_{k}}\right)\right), \widehat{S}_{2}\left(e_{i_{1}}, \ldots, e_{i_{k}}\right)\right\rangle \\
& =\sum_{i}\left\langle\widehat{S}_{1}\left(e_{i_{1}}, \ldots, e_{i_{k}}\right), \mathcal{R}^{P}\left(\widehat{S}_{2}\left(e_{i_{1}}, \ldots, e_{i_{k}}\right)\right)\right\rangle
\end{aligned}
$$

Thus, the claim follows.

Example 4. For vector fields and 1-forms, $\mathcal{R i c}^{P}$ reduces to the kind of usual Ricci curvature, $\operatorname{Ric}^{P}=\operatorname{Tr}_{1,3} R^{P}$. Let $X \in \mathfrak{X}_{M}$ and $\omega=X^{b}$ be the dual 1-form. Recall ([19], Proposition 3.6) that $\widehat{\omega}(Z)=Z \wedge X$. Thus, using Proposition 8 and (29), we obtain

$$
\begin{aligned}
& \left\langle\mathcal{R i c}^{P}(\omega), \omega\right\rangle=-\sum_{\alpha}\left\langle\mathcal{R}^{P}\left(\xi_{a}\right)\left(\xi_{a} \omega\right), \omega\right\rangle=\sum_{\alpha}\left\langle\xi_{a} \omega, \mathcal{R}^{P}\left(\xi_{a}\right) \omega\right\rangle \\
& =\sum_{i}\left\langle\widehat{\omega}\left(e_{i}\right), \mathcal{R}^{P}\left(\widehat{\omega}\left(e_{i}\right)\right)\right\rangle=\sum_{i}\left\langle\widehat{\omega}\left(e_{i}\right), \mathcal{R}^{P}\left(\widehat{\omega}\left(e_{i}\right)\right)\right\rangle \\
& =\sum_{i}\left\langle\mathcal{R}^{P}\left(e_{i} \wedge X\right), e_{i} \wedge X\right\rangle=\sum_{i}\left\langle R^{P}\left(e_{i}, X, X, e_{i}\right\rangle=\operatorname{Ric}^{P}(X, X) .\right.
\end{aligned}
$$

Now, we will extend ([15], Corollary 9.3.4).

Proposition 10. If $\left\langle\mathcal{R}^{P}(S), S\right\rangle \geq 0$ for any $(0, k)$-tensor $S$, then $\left\langle\mathcal{R i c}^{P}(S), S\right\rangle \geq 0$. Moreover, if $\left\langle\mathcal{R}^{P}(S), S\right\rangle \geq k\|S\|^{2}$ for any $(0, k)$-tensor $S$, where $k<0$, then $\left\langle\mathcal{R i c}^{P}(S), S\right\rangle \geq k C\|S\|^{2}$, where a constant $C$ depends only on the type of $S$.

Proof. Using (35) and a local orthonormal base $\left\{\xi_{\alpha}\right\}$ of $\Lambda^{2}(T M)$ such that $\mathcal{R}\left(\xi_{\alpha}\right)=\lambda_{\alpha} \xi_{\alpha}$, we get

$$
\left\langle\mathcal{R i c}^{P}(S), S\right\rangle=\sum_{\alpha} \lambda_{\alpha}\left\langle\mathcal{P}\left(\xi_{\alpha} S\right), \xi_{\alpha} S\right\rangle=\sum_{\alpha}\left\langle\mathcal{P}\left(\xi_{\alpha} S\right), \mathcal{R}\left(\xi_{\alpha} S\right)\right\rangle=\sum_{\alpha}\left\langle\mathcal{R}^{P}\left(\xi_{\alpha} S\right), \xi_{\alpha} S\right\rangle .
$$


If $\left\langle\mathcal{R}^{P}\left(\xi_{\alpha} S\right), \xi_{\alpha} S\right\rangle \geq 0$ for all $\alpha$, then $\left\langle\mathcal{R i c}^{P}(S), S\right\rangle \geq 0$, and the first claim follows. There is a constant $C>0$ depending only on the type of the tensor and $\operatorname{dim} M$ such that

$$
C\|S\|^{2} \geq \sum_{\alpha}\left\|\xi_{\alpha} S\right\|^{2}
$$

see ([15], Corollary 9.3.4). By conditions, $\left\langle\mathcal{R}^{P}\left(\xi_{\alpha} S\right), \xi_{\alpha} S\right\rangle \geq k\left\|\xi_{\alpha} S\right\|^{2}$ for all $\alpha$. The above, for $k<0$, yields $\left\langle\mathcal{R}^{P}\left(\xi_{\alpha} S\right), \xi_{\alpha} S\right\rangle \geq k C\|S\|^{2}$ - thus the second claim.

Theorem 3. Let $\left\langle\mathcal{R}^{P}(\omega), \omega\right\rangle \geq 0$ for any $k$-form $\omega$ on an open complete $(M, g)$. If $\left\|P P^{*} \nabla^{P}\left(\|\omega\|^{2}\right)\right\| \in$ $L^{1}(M, g)$ hold for a P-harmonic form $\omega$, then $\omega$ is P-parallel.

Proof. By conditions and Proposition 10, $\left\langle\mathcal{R i c}^{P}(\omega), \omega\right\rangle \geq 0$. By the modified Bochner-Weitzenböck formula (33) with $S=\omega$, since $\Delta_{H}^{P} \omega=0$, we obtain $\bar{\Delta}^{P}\left(\|\omega\|^{2}\right) \geq 0$. By Lemma 2 with $X=\nabla^{P}\left(\|\omega\|^{2}\right)$, we get $\bar{\Delta}^{P}\left(\|\omega\|^{2}\right)=0$. Hence, by (33) again, $\nabla^{P} \omega=0$.

Corollary 1 (For $P=\mathrm{id}_{T M}$, see Theorem 3.3 in [19]). If $\left\langle\mathcal{R}^{P}(\omega), \omega\right\rangle \geq 0$ for any $k$-form $\omega$ on a closed $(M, g)$, then any $P$-harmonic differential form on $M$ is P-parallel.

Proof. We give a short proof. By conditions and Proposition $10,\left\langle\operatorname{Ric}^{P}(\omega), \omega\right\rangle \geq 0$. By (31), since $\Delta_{H}^{P} \omega=0$, we obtain $\left\langle\bar{\Delta}^{P} \omega, \omega\right\rangle \leq 0$. By Proposition 2 with $S=\omega$, we get $\nabla^{P} \omega=0$.

Notice that, if the distribution $P(T M)$ in Theorem 3 and Corollary 1 is bracket-generating, then $\|\omega\|=$ const.

Remark 3. One may define also a linear operator $\overline{\mathcal{R}}^{P}=\mathcal{P}^{*} \circ \mathcal{R} \circ \mathcal{P}$ on $\Lambda^{2}(T M)$ that is

$$
\overline{\mathcal{R}}^{P}(X \wedge Y)=\mathcal{P}^{*} \circ \mathcal{R}^{P}(X \wedge Y)=\mathcal{P}^{*} \circ \mathcal{R} \circ \mathcal{P}(X \wedge Y) .
$$

Then, using known properties of $\mathcal{R}$ and Proposition 6, we have

$$
\left\langle\overline{\mathcal{R}}^{P}(X \wedge Y), Z \wedge W\right\rangle=\langle\mathcal{R}(P X \wedge P Y), \mathcal{P}(Z \wedge W)\rangle=R(P X, P Y, P W, P Z) .
$$

The $\overline{\mathcal{R}}^{P}$ on $\Lambda^{2}(T M)$ is self-adjoint (we can also consider it as a bilinear form on $\Lambda^{2}(T M)$ ):

$$
\begin{aligned}
\left\langle\overline{\mathcal{R}}^{P}(X \wedge Y), Z \wedge W\right\rangle & =R(P X, P Y, P W, P Z)=R(P Z, P W, P Y, P X) \\
& =\langle\mathcal{R}(P Z \wedge P W), P X \wedge P Y\rangle=\left\langle\overline{\mathcal{R}}^{P}(Z \wedge W), X \wedge Y\right\rangle .
\end{aligned}
$$

One may define a map $\overline{\mathcal{R}}^{P}(\xi): \mathcal{X}_{M} \rightarrow \mathcal{X}_{M}$ for $\xi \in \Lambda^{2}(T M)$

$$
\left\langle\overline{\mathcal{R}}^{P}(X \wedge Y) Z, W\right\rangle=\left\langle\overline{\mathcal{R}}^{P}(X \wedge Y), W \wedge Z\right\rangle=R(P X, P Y, P Z, P W) .
$$

Unlike $\mathcal{R}^{P}$, the map $\overline{\mathcal{R}}^{P}$ is skew-symmetric: $\left\langle\overline{\mathcal{R}}^{P}(\xi) W, Z\right\rangle=-\left\langle\overline{\mathcal{R}}^{P}(\xi) Z, W\right\rangle$.

It allows similar to $\mathcal{R}^{P}$ (in Lemma 4) decomposition $\overline{\mathcal{R}}^{P}(X \wedge Y)=-\sum_{\alpha}\left\langle\overline{\mathcal{R}}^{P}\left(\xi_{\alpha}\right) X, Y\right\rangle \xi_{\alpha}$.

Next, we define another Weitzenböck type curvature operator on a $(0, k)$-tensor $S$,

$$
\overline{\mathcal{R} i c}^{P}(S)\left(X_{1}, \ldots, X_{k}\right)=\sum_{i, \alpha}\left(\overline{\mathcal{R}}^{P}\left(e_{i} \wedge X_{\alpha}\right) S\right)(\underbrace{X_{1}, \ldots, e_{i}}_{\alpha}, \ldots, X_{k}),
$$

which unlike $\operatorname{Ric}^{P}$ is self-adjoint, and rewrite it as $\overline{\mathcal{R} i c}^{P}(S)=-\sum_{\alpha} \overline{\mathcal{R}}^{P}\left(\xi_{a}\right)\left(\xi_{a} S\right)$, see Proposition 8. Thus, if $S_{1}$ and $S_{2}$ are $(0, k)$-tensors on $(M, g)$, then (compare with Corollary 9)

$$
\left\langle\overline{\mathcal{R i c}}^{P}\left(S_{1}\right), S_{2}\right\rangle=\left\langle\overline{\mathcal{R}}^{P}\left(\widehat{S}_{1}\right), \widehat{S}_{2}\right\rangle .
$$


Similarly to Proposition 10, we get the following: If $\overline{\mathcal{R}}^{P} \geq 0$, then $\overline{\mathcal{R} \text { ic }} P \geq 0$; moreover, if $\overline{\mathcal{R}}^{P} \geq k$, where $k\left\langle 0\right.$, then $\left\langle\overline{\mathcal{R i c}}^{P}(S), S\right\rangle \geq k C\|S\|^{2}$, where a constant $C$ depends only on the type of the tensor $S$. We conclude by the following analog of Corollary 1: If $\overline{\mathcal{R}}^{P} \geq 0$ on a closed $(M, g)$, then all P-harmonic forms on $M$ are P-parallel. We omit results similar to Theorem 3.

Author Contributions: Investigation, P.P., V.R. and S.S. All authors have read and agreed to the published version of the manuscript.

Funding: This research received no external funding.

Conflicts of Interest: The authors declare no conflict of interest.

\section{Appendix A. Proof of Proposition 6}

By (23), we have for further use $P \nabla_{P X} Y=P \nabla_{X}^{P} Y=\nabla_{X}^{P} P Y=\nabla_{P X} P Y$.

1. We calculate

$$
\nabla_{X}^{P} \nabla_{Y}^{P} f-\nabla_{Y}^{P} \nabla_{X}^{P} f-\nabla_{[X, Y]_{P}}^{P} f=P X(P Y(f))-P Y(P X(f))-\left(P[X, Y]_{P}\right) f=\mathfrak{D}^{P}(X, Y) f=0 .
$$

Then,

$$
\begin{aligned}
& \left\langle R_{X, Y}^{P} Z, W\right\rangle=\left\langle\nabla_{X}^{P} \nabla_{Y}^{P} Z-\nabla_{Y}^{P} \nabla_{X}^{P} Z-\nabla_{[X, Y]_{P}}^{P} Z, W\right\rangle \\
& =\left\langle\nabla_{P X} \nabla_{P Y} Z-\nabla_{P Y} \nabla_{P X} Z-\nabla_{P[X, Y]_{P}} Z, W\right\rangle \\
& =\left\langle\nabla_{P X} \nabla_{P Y} Z-\nabla_{P Y} \nabla_{P X} Z-\nabla_{[P X, P Y]} Z, W\right\rangle=\left\langle R_{P X, P Y} Z, W\right\rangle .
\end{aligned}
$$

Similarly, $\left\langle R_{X, Y}^{P} W, Z\right\rangle=\left\langle R_{P X, P Y} W, Z\right\rangle$. Thus,

$$
\begin{aligned}
& \left(R_{X, Y}^{P} g\right)(Z, W)=R_{X, Y}^{P}\langle Z, W\rangle-\left\langle R_{X, Y}^{P} Z, W\right\rangle-\left\langle Z, R_{X, Y}^{P} W\right\rangle \\
& =-\left\langle R_{P X, P Y} Z, W\right\rangle-\left\langle Z, R_{P X, P Y} W\right\rangle=\left(R_{P X, P Y} g\right)(Z, W)=0 .
\end{aligned}
$$

2. Since $P[X, Y]_{P}=[P X, P Y]$, we have

$$
\begin{aligned}
R_{X, Y}^{P} Z & =\nabla_{P X} \nabla_{P Y} Z-\nabla_{P Y} \nabla_{P X} Z-\nabla_{P[X, Y]_{P}} Z=R_{P X, P Y} Z, \\
\left(R_{X, Y}^{P} \omega\right) Z & =R_{X, Y}^{P}(\omega(Z))-\omega\left(R_{X, Y}^{P} Z\right)=-\omega\left(R_{P X, P Y} Z\right) .
\end{aligned}
$$

3. This follows from 1 and 2 , since the actions of $R_{X, Y}^{P}$ and $R_{P X, P Y}$ on generators (functions, vector and covector fields) are the same.

4. We have

$$
\begin{aligned}
P R_{X, Y}^{P} Z & =P\left(\nabla_{X}^{P} \nabla_{Y}^{P} Z-\nabla_{Y}^{P} \nabla_{X}^{P} Z-\nabla_{[X, Y]_{P}}^{P} Z\right)=\nabla_{X}^{P} P \nabla_{Y}^{P} Z-\nabla_{Y}^{P} P \nabla_{X}^{P} Z-\nabla_{[X, Y]_{P}}^{P} P Z \\
& =\nabla_{X}^{P} \nabla_{Y}^{P} P Z-\nabla_{Y}^{P} \nabla_{X}^{P} P Z-\nabla_{[X, Y]_{P}}^{P} P Z=R_{X, Y}^{P} P Z .
\end{aligned}
$$

5. We have, using 4 ,

$$
\begin{aligned}
& R^{P^{*} P}(X, Y, Z, W)=\left\langle R_{X, Y}^{P} Z,\left(P^{*} P\right) W\right\rangle=\left\langle P R_{X, Y}^{P} Z, P W\right\rangle \\
& =\left\langle R_{X, Y}^{P} P Z, P W\right\rangle=R^{P}(X, Y, P Z, P W)=R(P X, P Y, P Z, P W) .
\end{aligned}
$$

6. Since $R_{X, Y}^{P} Z=-R_{Y, X}^{P} Z$, the first equality follows. For the second one, we use 1 :

$$
0=R_{X, Y}^{P} g(Z, Z)=2\left\langle R_{X, Y}^{P} Z, Z\right\rangle ;
$$

thus, we obtain 6 by polarizing the identity $\left\langle R_{X, Y}^{P}(Z+W), Z+W\right\rangle=0$.

7. By $\nabla g=0$, we get $R_{X, Y}^{P} g=0$, then $\left\langle R_{X, Y}^{P} Z, W\right\rangle+\left\langle Z, R_{X, Y}^{P} W\right\rangle=0$. 


\section{References}

1. Bejancu, A.; Farran, H. Foliations and Geometric Structures; Springer: London, UK, 2006.

2. Blair, D. Riemannian Geometry of Contact and Symplectic Manifolds; Springer: London, UK, 2010.

3. Bullo, F.; Lewis, A.D. Geometric Control of Mechanical Systems: Modeling, Analysis, and Design for Simple Mechanical Control Systems; Texts in Applied Math. 49; Springer: London, UK, 2005.

4. Calin, O.; Chang, D.-C. Sub-Riemannian Geometry. General Theory and Examples; Encyclopedia of Mathematics and Its Applications, 126; Cambridge University Press: Cambridge, UK, 2009.

5. Vaisman, I. Lectures on the Geometry of Poisson Manifolds; Progress in Math., 118; Springer: London, UK, 1994.

6. Mackenzie, K.C.H. General Theory of Lie Groupoids and Lie Algebroids; London Math. Soc. Lecture Note Series; Cambridge University Press: Cambridge, UK, 2005; Volume 213.

7. Popescu, M.; Popescu, P. Almost Lie Algebroids and Characteristic Classes. Symmetry Integr. Geom.-Methods Appl. 2019, 15, 021. [CrossRef]

8. Popescu, P. Almost Lie structures, derivations and R-curvature on relative tangent spaces. Rev. Roum. Math. Pures Appl. 1992, 37, 779-789.

9. Alexandrino, M.M.; Briquet, R.; Toeben, D. Progress in the theory of singular Riemannian foliations. Differ. Geom. Appl. 2013, 31, 248-267. [CrossRef]

10. Popescu, P.; Rovenski, V. An integral formula for singular distributions. Results Math. 2019, 75, 18. [CrossRef]

11. Gray, A. Pseudo-Riemannian almost product manifolds and submersions. J. Math. Mech. 1967, 16, 715-737.

12. Stepanov, S.E. Riemannian almost product manifolds and submersions. Part I. J. Math. Sci. 2000, 99, 1788-1810. [CrossRef]

13. Stepanov, S.E.; Tsyganok, I.I. A remark on the mixed scalar curvature of a manifold with two orthogonal totally umbilical distributions. Adv. Geom. 2019, 19, 291-296. [CrossRef]

14. Popescu, P.; Popescu, M. On singular non-holonomic geometry. Balkan J. Geom. Appl. 2013, 18, 58-68.

15. Petersen, P. Riemannian Geometry, 3rd ed.; Springer: London, UK, 2016.

16. Jost, J. Riemannian Geometry and Geometric Analysis, 7th ed.; Springer: London, UK, 2017.

17. Rovenski, V.; Stepanov, S.E.; Tsyganok, I. Geometry in the large of the kernel of Lichnerowicz Laplacians and its applications. arXiv 2019, arXiv:1903.10230.

18. Rosenberg, S. Semigroup domination and vanishing theorems. In Geometry of Random Motion (Ithaca, N.Y., 1987); volume 73 of Contemp. Math.; American Mathematical Society: Providence, RI, USA, 1988; pp. 287-302.

19. Petersen, P. Demystifying the Weitzenböck Curvature Operator. Available online: http://www.math.ucla. edu/ petersen/ (accessed on 29 February 2020).

20. Popescu, M.; Popescu, P. Geometrical objects on anchored vector bundles. In Lie Algebroids and Related Topics in Differential Geometry; Kubarski, J., Urbanski, P., Wolak, R., Eds.; Banach Center Publ.: Będlewo, Poland, 2001, Volume 54, pp. 217-233.

21. Grützmann, M.; Hu, X. Cohomology for almost Lie algebroids. arXiv 2012, arXiv:1206.5466, 20p.

22. Yano, K. On a Structure $f$ Satisfying $f+f^{3}=0$; Technical Report, No. 12; University of Washington: Washington, DC, USA, 1961.

23. Goldberg, S.I.; Yano, K. On normal globally framed $f$-manifolds. Tohoku Math. J. 1970, 22, 362-370. [CrossRef]

24. Gray, A. The structure of nearly Kähler manifolds. Math. Ann. 1976, 223, 233-248. [CrossRef]

25. Balashchenko, V. Invariant $f$-structures on naturally reductive homogeneous spaces. Russ. Math. (Iz. VUZ) 2008, 52, 1-12. [CrossRef]

26. Endo, H. Some remarks of nearly cosymplectic manifolds of constant $\phi$-sectional curvature. Tensor (N.S.) 2007, 68, 204-221.

27. Cappelletti-Montano, B.; De Nicola, A.; Dileo, G.; Yudin, I. Nearly Sasakian manifolds revisited. Complex Manifolds 2019, 6, 320-334. [CrossRef]

28. Chow, W.-L. Über Systeme von linearen partiellen Differentialgleichungen erster Ordnung. Math. Ann. 1939, 117, 98-105.

(C) 2020 by the authors. Licensee MDPI, Basel, Switzerland. This article is an open access article distributed under the terms and conditions of the Creative Commons Attribution (CC BY) license (http://creativecommons.org/licenses/by/4.0/). 